\title{
Trigonal warping and anisotropic band splitting in monolayer graphene due to Rashba spin-orbit coupling
}

\author{
P. Rakyta, ${ }^{1}$ A. Kormányos, ${ }^{2, *}$ and J. Cserti ${ }^{1}$ \\ ${ }^{1}$ Department of Physics of Complex Systems, Eötvös University, Pázmány Péter sétány 1/A, H-1117 Budapest, Hungary \\ ${ }^{2}$ Department of Physics, Lancaster University, Lancaster LA1 4YB, United Kingdom
}

(Received 14 June 2010; published 7 September 2010)

\begin{abstract}
We study the electronic band structure of monolayer graphene when Rashba spin-orbit coupling is present. We show that if the Rashba spin-orbit coupling is stronger than the intrinsic spin-orbit coupling, the low-energy bands undergo trigonal-warping deformation and that for energies smaller than the Lifshitz energy, the Fermi circle breaks up into separate parts. The effect is very similar to what happens in bilayer graphene at low energies. We discuss the possible experimental implications, such as threefold increase in the minimal conductivity for low electron densities, anisotropic, wave-number-dependent spin splitting of the bands, and the spin-polarization structure.
\end{abstract}

DOI: 10.1103/PhysRevB.82.113405

PACS number(s): 73.22.Pr, 73.23.Ad

Following the pioneering work of Kane and Mele ${ }^{1}$ where a novel type of quantum spin-Hall effect was predicted, there has been a significant interest in studying the spin-orbit (SO) coupling in graphene. Generally, the SO interaction in graphene can have intrinsic or extrinsic origin. Densityfunctional and other calculations indicate ${ }^{2-4}$ that the intrinsic SO (ISO) coupling is in the range of $1-50 \mu \mathrm{eV}$ and therefore it could only be probed at very low temperatures. The SO interaction can also be induced externally, by impurities, ${ }^{5}$ by external electric field perpendicular to the graphene sheet or it can be due to the substrate. ${ }^{6}$ The latter two mechanism can introduce Rashba-type SO (RSO) interaction, which will be in the focus of our interest in this Brief Report. Many experimental implications of both intrinsic and extrinsic SO coupling in graphene have been considered in the literature. ${ }^{1,5,7-11}$ The first experimental report of spin-split bands due to a magnetic substrate was by Dedkov et al. ${ }^{12}$ Shortly after Ref. 12 the experiment of Varykhalov et al. ${ }^{6}$ showed that it was possible to fabricate a quasi-free-standing graphene on $\mathrm{Ni}(111)$ surface with a single layer of intercalated gold atoms between the graphene layer and the Ni surface. A spin-orbit interaction-induced band splitting of $\approx 13 \mathrm{meV}$ was measured, which is two-three orders of magnitude larger than the predictions for ISO..$^{2-4}$ The spin-orbit coupling was identified as RSO and the large splitting was attributed to the high nuclear charge of the intercalating gold atoms. Very recently, Gierz et al. have also observed a large and anisotropic spin splitting in graphene samples on $\mathrm{SiC}$ substrate.

The microscopic Hamiltonian describing the RSO coupling in graphene, shown in Eq. (1b), was introduced by Kane and Mele in Ref. 13 and its properties were subsequently studied in, e.g., Refs. 7, 8, 14, and 15 . Here we report on an interesting feature of the low-energy spectrum of this Hamiltonian which has attracted little attention so far but which can be important in systems where RSO coupling is much stronger than ISO. Examples for such systems appear to be the ones studied in the experiments of Varykhalov et al. ${ }^{6}$ and Gierz et al. ${ }^{16}$ We show that close to the $\mathbf{K}$ and $\mathbf{K}^{\prime}$ points of the graphene's Brillouin zone the Hamiltonian of monolayer graphene with RSO interaction is in good ap- proximation equivalent to the Hamiltonian of bilayer graphene ${ }^{17}$ and that RSO interaction in monolayer graphene leads to trigonal warping (TW) of the energy bands. This equivalence of the Hamiltonians may seem surprising since the two physical systems are very different. We show numerically that as long as RSO interaction is stronger than ISO, it affects the band structure in two important ways: (i) TW changes the topology of the low-energy bands at the $\mathbf{K}$ $\left(\mathbf{K}^{\prime}\right)$ points which may be detected as a treefold increase in the minimal conductivity of the sample at very low electron densities. (ii) RSO is also manifested through the nonisotropic spin splitting of the bands and in particular, through the nonconstant band splitting at the $\mathbf{K}\left(\mathbf{K}^{\prime}\right)$ points.

Tight-binding model. We start our discussion by considering the tight-binding (TB) Hamiltonian of monolayer graphene which includes RSO coupling ${ }^{13}$ as well. It is given by $H=H_{0}+H_{R}$, where

$$
H_{0}=-\gamma_{0} \sum_{\langle i, j\rangle, \sigma}\left(a_{i \sigma}^{\dagger} b_{j \sigma}+\text { H.c. }\right) \text {. }
$$

Here $\gamma_{0}$ is the hopping amplitude between nearest-neighbor atoms, $a_{i \sigma}^{\dagger}\left(a_{i \sigma}\right)$ creates (annihilates) an electron in the $i$ th unit cell with spin $\sigma$ on sublattice $A$ while $b_{j \sigma}^{\dagger}\left(b_{j \sigma}\right)$ has the same effect on sublattice $B$. The sum is taken over nearestneighbor atoms $\langle i, j\rangle$ and H.c. stands for Hermitian conjugate. The Hamiltonian $H_{R}$ describes the Rashba spin-orbit coupling and it reads ${ }^{13}$

$$
H_{R}=i \lambda_{R} \sum_{\langle i, j\rangle, \mu, \nu}\left[a_{i \mu}^{\dagger}\left(\mathbf{s}_{\mu \nu} \times \hat{\mathbf{d}}_{\langle i, j\rangle}\right)_{z} b_{j \nu}-\text { H.c. }\right]
$$

where $\mathbf{s}=\left(s_{x}, s_{y}, s_{z}\right)$ are the Pauli matrices representing the electron-spin operator and $\mu, \nu=1,2$ denote the $\mu \nu$ matrix elements of the Pauli matrices. Moreover, $\hat{\mathbf{d}}_{\langle i, j\rangle}=\mathbf{d}_{\langle i, j\rangle} / d$ are unit vectors, where $\mathbf{d}_{\langle i, j\rangle}$ points from atom $j$ to its nearest neighbors $i$ and $d=\left|\mathbf{d}_{\langle i, j\rangle}\right\rangle$. The strength of the spin-orbit coupling is denoted by $\lambda_{R}$ which may arise due to a perpendicular electric field or interaction with the substrate. 
Using the operator $\alpha_{\mathbf{q} \sigma}^{\dagger}=\frac{1}{\sqrt{N}} \sum_{i} e^{i \mathbf{q} \mathbf{R}_{i}} a_{i \sigma}^{\dagger}$, where $\mathbf{R}_{i}$ is the Bravais vector of the $i$ th unit cell and $\mathbf{q}$ lies in the first Brillouin zone (and similarly, introducing $\beta_{\mathbf{q} \sigma}^{\dagger}$ acting on sublattice $B$ ) it is easy to find that

$$
\begin{gathered}
H_{0}=-\gamma_{0} \sum_{\mathbf{q}, \sigma}\left[f(\mathbf{q}) \alpha_{\mathbf{q} \sigma}^{\dagger} \beta_{\mathbf{q} \sigma}+\text { H.c. }\right], \\
H_{R}=i \lambda_{R} \sum_{\mathbf{q}, \mu \nu}\left\{\alpha_{\mathbf{q} \mu}^{\dagger}\left[\mathbf{s}_{\mu \nu} \times \mathbf{D}(\mathbf{q})\right]_{z} \beta_{\mathbf{q} \nu}-\text { H.c. }\right\},
\end{gathered}
$$

where

$$
f(\mathbf{q})=\sum_{j=1}^{3} e^{-i \mathbf{q} \mathbf{a}_{j}} \quad \text { and } \quad \mathbf{D}(\mathbf{q})=-\sum_{j=1}^{3} \hat{\mathbf{d}}_{j} e^{-i \mathbf{q} \mathbf{a}_{j}} .
$$

Here $\mathbf{a}_{1}$ and $\mathbf{a}_{2}$ are the lattice vectors of graphene and it was convenient to introduce the vector $\mathbf{a}_{3}=0$. Finally, the matrix representation of the Hamiltonian $H=H_{0}+H_{R}$ acting on wave function $\Psi=\left(\Psi_{A \uparrow}, \Psi_{B \uparrow}, \Psi_{A \downarrow}, \Psi_{B \downarrow}\right)$ can be written as

$$
H(\mathbf{q})=\left(\begin{array}{cccc}
0 & -\gamma_{0} f(\mathbf{q}) & 0 & -\lambda_{R} D_{+}(\mathbf{q}) \\
-\gamma_{0} f^{*}(\mathbf{q}) & 0 & -\lambda_{R} D_{-}^{*}(\mathbf{q}) & 0 \\
0 & -\lambda_{R} D_{-}(\mathbf{q}) & 0 & -\gamma_{0} f(\mathbf{q}) \\
-\lambda_{R} D_{+}^{*}(\mathbf{q}) & 0 & -\gamma_{0} f^{*}(\mathbf{q}) & 0
\end{array}\right) \text {, }
$$

where $D_{ \pm}(\mathbf{q})= \pm D_{x}(\mathbf{q})-i D_{y}(\mathbf{q})$. This TB Hamiltonian can be used for calculations in the whole Brillouin zone (BZ). As it has been shown in Ref. 14, the Hamiltonian in Eq. (3) preserves the particle-hole symmetry therefore in the following we will focus on the conduction bands only. One finds that the electron and hole bands touch at the $\mathbf{K}$ and $\mathbf{K}^{\prime}$ points of the Brillouin zone [here $\mathbf{K}=\left(2 \mathbf{b}_{2}+\mathbf{b}_{1}\right) / 3$ and $\mathbf{K}^{\prime}=\left(2 \mathbf{b}_{1}\right.$ $\left.+\mathbf{b}_{2}\right) / 3$, where $\mathbf{b}_{1}$ and $\mathbf{b}_{2}$ are the reciprocal lattice vectors of the graphene sheet]. Next, it will prove instructive to derive a low-energy Hamiltonian which describes the excitations in the vicinity of the band-touching points.

Continuum model. We expand Hamiltonian (3) around the Dirac point $\mathbf{K}$ of the BZ in terms of $\mathbf{k}=\mathbf{q}-\mathbf{K}$ for $|\mathbf{k}| \approx 0$. Then one can perform a unitary transformation using the matrix $U=\left(\frac{i-1}{2} I_{2}+\frac{i+1}{2} \sigma_{z}\right) \otimes I_{2}$ (here $I_{2}$ is a $2 \times 2$ unit matrix) and take the inverse Fourier transformation corresponding to the replacement $\hbar \mathbf{k} \rightarrow \hat{\mathbf{p}}=-i \hbar \boldsymbol{\nabla}$ [here $\hat{\mathbf{p}}=\left(\hat{p}_{x}, \hat{p}_{y}\right)$ is the momentum operator]. One obtains finally the following Hamiltonian for monolayer graphene with RSO interaction:

$$
H_{K}=\left(\begin{array}{cccc}
0 & v_{F} \hat{p}_{-} & 0 & -v_{\lambda} \hat{p}_{+} \\
v_{F} \hat{p}_{+} & 0 & 3 i \lambda_{R} & 0 \\
0 & -3 i \lambda_{R} & 0 & v_{F} \hat{p}_{-} \\
-v_{\lambda} \hat{p}_{-} & 0 & v_{F} \hat{p}_{+} & 0
\end{array}\right) .
$$

Here $v_{F}=3 \gamma_{0} d /(2 \hbar), \quad v_{\lambda}=3 \lambda_{R} d /(2 \hbar), \quad$ and $\hat{p}_{ \pm}=\hat{p}_{x} \pm i \hat{p}_{y}$. Apart from a unitary transformation this Hamiltonian is the same as the Hamiltonian of bilayer graphene, if the asymmetry between the on-site energies of the bottom and top layers and the small hopping amplitude $\gamma_{4}$ can be neglected. ${ }^{17}$ This conclusion may seem surprising since the TB models of the two systems look rather different. As $v_{\lambda} \ll v_{F}$, when calculat-
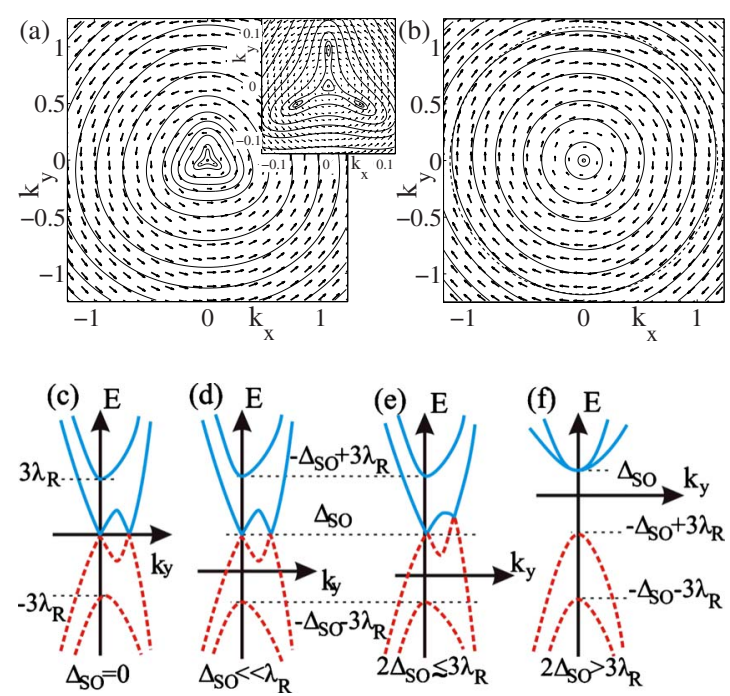

FIG. 1. (Color online) The $\mathbf{k}$ dependence and spin polarization of the energy bands: (a) $E_{1}^{+}(\mathbf{k})$ and (b) $E_{2}^{+}(\mathbf{k})$ around the $\mathbf{K}$ point for $\beta=0.034$. Solid lines show the constant-energy contours, the arrows indicate the direction of the spin at certain points in the BZ. The wave-vector components $k_{x}$ and $k_{y}$ are in units of $3 \lambda_{R} /\left(2 \hbar v_{F}\right)$. The inset (a) shows a closeup of $E_{1}^{+}(\mathbf{k})$ in the vicinity of the $\mathbf{K}\left(\mathbf{K}^{\prime}\right)$ point. The pocket structure has a $2 \pi / 3$ rotational symmetry and the distance of the pocket minima from the $\mathbf{K}\left(\mathbf{K}^{\prime}\right)$ point is $k_{\text {SO }}$ (see the text). The dashed line in (b) is a circle, it indicates that the isoenergy lines of $E_{2}^{+}(\mathbf{k})$ are also slightly distorted. [(c)-(f)] A cut through the energy bands for $k_{x}=0$ and (c) $\Delta_{\mathrm{SO}}=0$, (d) $\Delta_{\mathrm{SO}} \ll \lambda_{R}$, (e) $2 \Delta_{\mathrm{SO}} \lesssim 3 \lambda_{R}$, and (f) $2 \Delta_{\mathrm{SO}}>3 \lambda_{R}$. Solid (blue) lines denote electron bands while dashed (red) lines correspond to valance bands.

ing the band structure the matrix elements $\left(H_{K}\right)_{14}$ and $\left(H_{K}\right)_{41}$ are small compared to the matrix elements which are proportional to $v_{F}$ and one can question if it is important to keep them. Indeed, the theoretical treatment of RSO in Refs. 4, 7, 8 , and 11 is based on a Hamiltonian in which $\left(H_{K}\right)_{14}$ $=\left(H_{K}\right)_{41}=0$. Note, however, that these terms cause trigonal warping of the bands at low energy and they can therefore be important since TW results in the change in the topology of the energy bands near the Dirac point. This can be easily seen by recalling the band structure of bilayer graphene with TW (Ref. 17) or by looking at the plane-wave solutions of the Schrödinger equation $H_{K} \Psi(\mathbf{k})=E(\mathbf{k}) \Psi(\mathbf{k})$. The four eigenvalues of Hamiltonian (4) as a function of the wave number $\mathbf{k}=k(\cos \alpha, \sin \alpha)^{T}$ are given by

$$
E_{n}^{ \pm}(\mathbf{k})= \pm \hbar v_{F} \sqrt{\frac{1}{2}\left[k_{\lambda}^{2}+k^{2}\left(2+\beta^{2}\right)+(-1)^{n} \sqrt{\Upsilon}\right]},
$$

where $\beta=v_{\lambda} / v_{F}=\lambda_{R} / \gamma_{0}$ is the dimensionless strength of the spin-orbit coupling, $k_{\lambda}=2 \beta / d, n=1,2$, and

$$
\Upsilon=k_{\lambda}^{4}+2 k^{2} k_{\lambda}^{2}\left(2-\beta^{2}\right)+k^{4} \beta^{2}\left(4+\beta^{2}\right)-8 k^{3} k_{\lambda} \beta \sin (3 \alpha) .
$$

Figures 1(a) and 1(b) show the contour plot of the conduction bands $E_{1}^{+}(\mathbf{k})$ and $E_{2}^{+}(\mathbf{k})$. At $\mathbf{k}=\mathbf{0}$ the splitting of the bands is $3 \lambda_{R}$ [see Fig. 1(c)]. Similarly to bilayer graphene, the bands have a threefold rotational symmetry. For energies 
smaller than the Lifshitz energy $E_{L}=3 \lambda_{R} \beta^{2} /\left(4+\beta^{2}\right)$, the constant-energy lines of $E_{1}^{+}(\mathbf{k})$ are broken into four pockets, which can be referred to as one central and three leg parts [this is shown in the inset of Fig. 1(a)]. The eigenvalues $\pm E_{1}^{+}(\mathbf{k})$ become zero at the $\mathbf{K}$ point of the Brillouin zone, i.e., at $k=0$, and at the center of the three leg parts located at $k=k_{\mathrm{SO}}$ and $\alpha=-\pi / 6 ; \pi / 2 ; 7 \pi / 6$. The distance between the center of the leg parts and the Dirac point ( $\mathbf{K}$ point) in continuous model is $k_{\mathrm{SO}}=\beta^{2} \frac{2}{d}$, which is equal to $k_{\mathrm{SO}}$ in Ref. 14 for the experimentally relevant case of $\lambda_{R} \ll \gamma_{0}$. The authors of Ref. 14 have also noticed the three additional zero-energy points around the $\mathbf{K}$ point but it was not recognized that Hamiltonian (4) of monolayer graphene with RSO is equivalent to the Hamiltonian of bilayer graphene with TW. An important difference, however, between bilayer graphene and monolayer graphene with $\mathrm{RSO}$ is that the splitting $\Delta E(\mathbf{k})$ $=E_{2}^{+}(\mathbf{k})-E_{1}^{+}(\mathbf{k})$ of the two conduction bands is much smaller in the latter. This energy is in the range of $0.3-0.48 \mathrm{eV}$ (Ref. 18) for bilayer whereas a recent experiment ${ }^{6}$ shows that the splitting is about $13 \mathrm{meV}$ for RSO in monolayer graphene. Therefore in the latter system even for small electron densities states in both $E_{1}^{+}(\mathbf{k})$ and $E_{2}^{+}(\mathbf{k})$ would be occupied whereas in the case of bilayer graphene the occupation of the upper band can usually be neglected.

So far in our discussion we have completely neglected the intrinsic spin-orbit coupling ISO. As the Lifshitz energy $E_{L}$ itself is a very small energy scale, it is important to understand whether the trigonal warping and the pocket structure survive if a small, yet finite ISO is also present. To this end we have numerically calculated the band structure using the Hamiltonian $H=H_{0}+H_{R}+H_{\mathrm{SO}}$ with $H_{\mathrm{SO}}=\Delta_{\mathrm{SO}} \sigma_{z} s_{z}$ (see Ref. $1)$, where $\sigma_{z}=1(-1)$ describes state on sublattice $A(B)$. The results of the calculations are shown in Figs. 1(c)-1(f). For $\lambda_{R} \gg \Delta_{\text {SO }}$ the topology of the lower energy band around the band touching points is preserved [though the bands get a global shift, compare Figs. 1(c) and 1(d)]. Furthermore, if $2 \Delta_{\text {SO }} \lesssim 3 \lambda_{R}$, most of the characteristics of the RSO spectrum survive, but the minima of the leg pockets will be at slightly higher energy than the minimum of the central pocket [see Fig. 1(e)]. Finally, if the ISO splitting become larger than $3 \lambda_{R}$, the pocket structure vanishes completely [Fig. 1(f)]. Our findings in Figs. 1(c) and 1(d) are in good qualitative agreement with the density-functional calculations of Ref. 4 except that in the results of Ref. 4 the TW seems to be absent. This is not surprising, however, since for the weak Rashba splitting assumed there $\left(3 \lambda_{R} \approx 25 \mu \mathrm{eV}\right)$ trigonal warping is negligible. For stronger $\lambda_{R}$ values and if $\lambda_{R}$ $\gg \Delta_{\text {SO }}$, which is likely to be the case in the experiment of Refs. 6 and 16, TW is expected to be more important.

We now turn to discuss some of the experimental implications of our results and compare the predictions based on the Hamiltonian in Eqs. (4) and (3) to some of the results of recent experiments. One can immediately see that if the RSO coupling is much larger than the ISO, our results predict that due to the pocket structure shown in the inset of Fig. 1(a) for very low electron densities the minimal conductivity is three times larger than what one would obtain by neglecting TW. (For the relevant calculations for bilayer graphene see Refs. 19 and 20.)

Another way to study the TW experimentally would be to
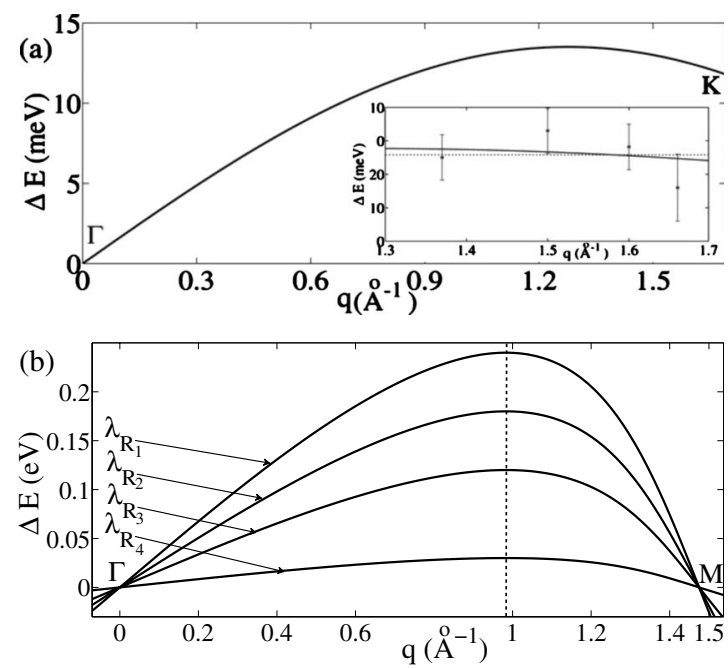

FIG. 2. (a) The spin splitting along the $\overline{\Gamma K}$ line of the BZ using the Hamiltonian of Eq. (3). The inset shows the comparison of the experimental data from Ref. 6 and the calculated band splitting with finite TW (solid line) and with no TW (constant dashed line). (b) The band splitting along $\overline{\Gamma M}$ for $\lambda_{R_{1}}=80 \mathrm{meV}, \lambda_{R_{2}}=60 \mathrm{meV}$, $\lambda_{R_{3}}=40 \mathrm{meV}$, and $\lambda_{R_{4}}=10 \mathrm{meV}$ obtained from Hamiltonian (3). Vertical dashed line indicates the minimum of the curves. The TB hopping parameter was $\gamma_{0}=3 \mathrm{eV}$.

scan the spin splitting of the bands across the BZ. By looking at Figs. 1(a) and 1(b) it is easy to see that the splitting is anisotropic around the $\mathbf{K}$ point and would reflect a threefold rotational symmetry. This is an important difference compared to the model used in Refs. 4, 7, 8, and 11. In contrast to the measurements of Ref. 16, in this model the splitting does not go to zero along the $\overline{\Gamma K}$ line, which may indicate a strong influence of the SiC substrate that the theoretical models considered so far do not capture. Since Hamiltonian (4) can only be applied around the $\mathbf{K}$ point, in order to explore a larger part of the BZ we also performed calculations using the TB model [see Eq. (3)]. The band splitting along the $\overline{\Gamma K}$ line assuming RSO coupling constant $3 \lambda_{R}=12 \mathrm{meV}$ is shown in Fig. 2(a). The most important feature to be observed is that the RSO splitting in the vicinity of the $\mathbf{K}$ point is not constant as predicted by the model which neglects TW but has a small curvature. This can also be seen in the inset of Fig. 2(a) which shows the comparison of the calculations and of the data from Fig. 4(c) of Ref. 6. The experimental data are consistent with the calculated curve, unfortunately the measurement resolution does not allow to make a definitive judgment as to whether the splitting is constant or not. In Fig. 2(b) we plot the spin splitting along the $\overline{\Gamma M}$ line in the $\mathrm{BZ}$ for various RSO coupling strengths. The maximum of the band splitting equals $3 \lambda_{R}$ and it is always attained at the same $k$ wave number, not depending on $\lambda_{R}$. It is interesting to note that the shape of these curves is very reminiscent of the measurements shown in Fig. 3(c) of Ref. 12, which were made on graphene on $\mathrm{Ni}$ substrate. The reason of the splitting in this system, however, appears to be a subject of debate. ${ }^{21,22}$

The spin and angle resolved photoemission spectroscopy (SARPES) technique ${ }^{23}$ cannot only probe the band structure but also the spin polarization of the bands. The results of 
Ref. 6 suggest that the spin polarization has circular symmetry around the $\mathbf{K}$ point and that the spin is polarized in clockwise (anticlockwise) directions in the two bands at the Fermi energy. Our calculations for the spin polarization of the energy bands $E_{1}^{+}(\mathbf{k})$ and $E_{2}^{+}(\mathbf{k})$ are in agreement with this experimental result [see in Figs. 1(a) and 1(b), respectively]. It might seem surprising that the spin polarization of the band $E_{1}^{+}(\mathbf{k})$ shows a simple rotational symmetry without any signs of TW observed in the spectrum. As we will demonstrate below, TW affects the spin polarization only in higher orders of $\mathbf{k}$. The calculation of spin polarization goes along the lines of a similar calculation in Ref. 7. The expectation value of the spin components on sublattice $A$ is given by $\mathbf{s}^{a}$ $=\hbar / 2\left(\sin \theta_{s}^{a} \cos \varphi_{s}^{a}, \sin \theta_{s}^{a} \sin \varphi_{s}^{a}, \cos \theta_{s}^{a}\right)^{T}$. We find that in the lowest orders it depends on $k=|\mathbf{k}|$ as

$$
\tan \theta_{n}^{a}(\mathbf{k})=(-1)^{n+1}\left(\frac{2 k}{k_{\lambda}}-\sin 3 \alpha \frac{k^{2} d}{k_{\lambda}}\right)
$$

and

$$
\varphi_{n}^{a}(\mathbf{k})=\alpha+(-1)^{n+1} \frac{\pi}{2}-\frac{\cos 3 \alpha}{2} k d,
$$

where the indexes $n=1,2$ correspond to energy eigenvalues $E_{1}^{+}(\mathbf{k})\left[\right.$ or $\left.E_{1}^{-}(\mathbf{k})\right]$ and $E_{2}^{+}(\mathbf{k})\left[\right.$ or $\left.E_{2}^{-}(\mathbf{k})\right]$, respectively. Similar calculations for sublattice $B$ yield $\theta_{n}^{b}(\mathbf{k})=\pi-\theta_{n}^{a}(\mathbf{k})$ and $\varphi_{n}^{b}(\mathbf{k})=\varphi_{n}^{a}(\mathbf{k})$. As one can see from Eqs. (6a) and (6b), TW does not play role in leading order of $k$. This explains the circular symmetry of the spin polarization in Figs. 1(a) and 1(b). Moreover, the component of the spin perpendicular to the graphene sheet points into opposite directions on sublattices $A$ and $B$. Therefore, as in Ref. 7, we find that the average spin $\mathbf{s}=\left(\mathbf{s}^{a}+\mathbf{s}^{b}\right) / 2$ is polarized in the plane of the graphene sheet, it is perpendicular to the wave vector $\mathbf{k}$ and its magnitude grows up from zero (at $k=0$ ) to $\hbar / 2$ further from the $\mathbf{K}$ point, see also in Figs. 1(a) and 1(b).

In summary, we have studied the RSO coupling in graphene monolayer. We have found that in case both ISO and RSO interactions are present, as long as the RSO coupling is stronger, the low-energy bands will show TW in the vicinity of the $\mathbf{K}$ point of the BZ. Our predictions for the spin splitting of the bands along certain high-symmetry directions in the BZ are consistent with the results of a recent experiment ${ }^{6}$ which measured the band splitting due to RSO interaction. Finally, we have demonstrated that the TW of the bands does not affect the spin polarization in the lowest order of the momentum therefore it shows rotational symmetry around the $\mathbf{K}$ point. These results might be relevant for other novel low-dimensional systems where RSO coupling is important. $^{23}$

We thank A. Varykhalov for helpful discussions and for providing the experimental data used in Fig. 2(a). P. R. and J. Cs. acknowledge the support of the Marie Curie ITN project NanoCTM (Grant No. FP7-PEOPLE-ITN-2008-234970) and the Hungarian Science Foundation OTKA under Contracts No. 75529 and No. 81492. A.K. was supported by EPSRC.

\footnotetext{
*a.kormanyos@lancaster.ac.uk

${ }^{1}$ C. L. Kane and E. J. Mele, Phys. Rev. Lett. 95, 226801 (2005).

${ }^{2}$ D. Huertas-Hernando, F. Guinea, and A. Brataas, Phys. Rev. B 74, 155426 (2006).

${ }^{3}$ H. Min, J. E. Hill, N. A. Sinitsyn, B. R. Sahu, L. Kleinman, and A. H. MacDonald, Phys. Rev. B 74, 165310 (2006); Y. Yao, F. Ye, X.-L. Qi, S.-C. Zhang, and Z. Fang, ibid. 75, 041401 (2007); J. C. Boettger and S. B. Trickey, ibid. 75, 121402 (2007).

${ }^{4}$ M. Gmitra, S. Konschuh, C. Ertler, C. Ambrosch-Draxl, and J. Fabian, Phys. Rev. B 80, 235431 (2009).

${ }^{5}$ A. H. Castro Neto and F. Guinea, Phys. Rev. Lett. 103, 026804 (2009).

${ }^{6}$ A. Varykhalov, J. Sánchez-Barriga, A. M. Shikin, C. Biswas, E. Vescovo, A. Rybkin, D. Marchenko, and O. Rader, Phys. Rev. Lett. 101, 157601 (2008).

${ }^{7}$ E. I. Rashba, Phys. Rev. B 79, 161409(R) (2009).

${ }^{8}$ F. Kuemmeth and E. I. Rashba, Phys. Rev. B 80, 241409(R) (2009).

${ }^{9}$ C. Ertler, S. Konschuh, M. Gmitra, and J. Fabian, Phys. Rev. B 80, 041405(R) (2009).

${ }^{10}$ D. Huertas-Hernando, F. Guinea, and A. Brataas, Phys. Rev. Lett. 103, 146801 (2009).

${ }^{11}$ P. Ingenhoven, J. Z. Bernád, U. Zülicke, and R. Egger, Phys.
}

Rev. B 81, 035421 (2010).

${ }^{12}$ Yu. S. Dedkov, M. Fonin, U. Rüdiger, and C. Laubschat, Phys. Rev. Lett. 100, 107602 (2008).

${ }^{13}$ C. L. Kane and E. J. Mele, Phys. Rev. Lett. 95, 146802 (2005).

${ }^{14}$ M. Zarea and N. Sandler, Phys. Rev. B 79, 165442 (2009).

${ }^{15}$ Z. Wang, N. Hao, and P. Zhang, Phys. Rev. B 80, 115420 (2009).

${ }^{16}$ I. Gierz, J. Dil, F. Meier, B. Slomski, J. Osterwalder, J. Henk, R. Winkler, C. Ast, and K. Kern, arXiv:1004.1573 (unpublished).

${ }^{17}$ E. McCann and V. I. Fal'ko, Phys. Rev. Lett. 96, 086805 (2006).

${ }^{18}$ L. M. Malard, J. Nilsson, D. C. Elias, J. C. Brant, F. Plentz, E. S. Alves, A. H. Castro Neto, and M. A. Pimenta, Phys. Rev. B 76, 201401 (2007); T. Ohta, A. Bostwick, J. L. McChesney, T. Seyller, K. Horn, and E. Rotenberg, Phys. Rev. Lett. 98, 206802 (2007)

${ }^{19}$ J. Cserti, A. Csordás, and G. Dávid, Phys. Rev. Lett. 99, 066802 (2007).

${ }^{20}$ A. G. Moghaddam and M. Zareyan, Phys. Rev. B 79, 073401 (2009).

${ }^{21}$ O. Rader, A. Varykhalov, J. Sánchez-Barriga, D. Marchenko, A. Rybkin, and A. M. Shikin, Phys. Rev. Lett. 102, 057602 (2009).

${ }^{22}$ A. Varykhalov and O. Rader, Phys. Rev. B 80, 035437 (2009).

${ }^{23}$ J. H. Dil, J. Phys.: Condens. Matter 21, 403001 (2009). 